\title{
New Eulerian numbers of type $D$
}

\author{
Anna Borowiec Wojciech Młotkowski* \\ Instytut Matematyczny \\ Uniwersytet Wrocławski \\ Plac Grunwaldzki 2/4 \\ 50-384 Wrocław, Poland \\ anna.malgorzata.borowiec@gmail.com \\ mlotkow@math.uni.wroc.pl
}

Submitted: Aug 25, 2015; Accepted: Feb 12, 2016; Published: Feb 19, 2016

Mathematics Subject Classifications: 05A05, 20B35

\begin{abstract}
We introduce a new array of type $D$ Eulerian numbers, different from that studied by Brenti, Chow and Hyatt. We find in particular the recurrence relation, Worpitzky formula and the generating function. We also find the probability distributions whose moments are Eulerian polynomials of type $A, B$ and $D$.
\end{abstract}

Keywords: Eulerian numbers, signed permutations, moments of a probability measure.

\section{Introduction}

For a sequence $\left(a_{1}, a_{2}, \ldots, a_{s}\right), a_{i} \in \mathbb{R}$, the number of descents is defined as the cardinality of the set

$$
\left\{i \in\{1,2, \ldots, s-1\}: a_{i}>a_{i+1}\right\} .
$$

The aim of this paper is to present a new array of type $D$ Eulerian numbers, different from that studied by Brenti [5], Chow [6] and Hyatt [9]. They define $D_{n, k}$ as the number of elements $\sigma$ in the group $\mathcal{D}_{n}$ (see Section 4 for the definition) such that the sequence $(-\sigma(2), \sigma(1), \sigma(2), \ldots, \sigma(n))$ has $k$ descents $(A 066094$ in OEIS [10]). For $0 \leqslant n \leqslant 4$ these numbers look as follows:

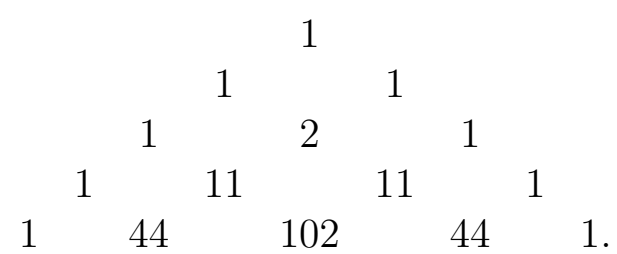

*supported by the Polish National Science Center grant No. 2012/05/B/ST1/00626. 
In this paper we define type $D$ Eulerian numbers $D(n, k)$ by counting descents in the sequence $(0, \sigma(1), \sigma(2), \ldots, \sigma(n))$, like for type $B$. First we find the basic recurrence relations, which involve the numbers $D(n, k)$ together with $\widetilde{D}(n, k):=B(n, k)-D(n, k)$ (Theorem 4.2). Then we prove a simple relation between the numbers $D(n, k)$ and $\widetilde{D}(n, k)$ (Proposition 4.3) and between $D(n, k)$ and $B(n, k)$ (Corollary 4.4). Next we derive new recurrence relations for $D(n, k)$ and $\widetilde{D}(n, k)$ independently (Proposition 4.5$)$. We find Worpitzky type formulas and the generating function for the numbers $D(n, k), \widetilde{D}(n, k)$ and prove the real-rootedness property for the corresponding polynomials $P_{n}^{\mathrm{D}}(t), P_{n}^{\widetilde{\mathrm{D}}}(t)$.

We also prove that for $t \geqslant 0$ the sequences $\left\{P_{n}^{\mathrm{A}}(t)\right\}_{n=0}^{\infty},\left\{P_{n}^{\mathrm{B}}(t)\right\}_{n=0}^{\infty}$ and $\left\{P_{n}^{\mathrm{D}}(t)\right\}_{n=0}^{\infty}$ are positive definite, where $P_{n}^{\mathrm{A}}(t), P_{n}^{\mathrm{B}}(t)$ and $P_{n}^{\mathrm{D}}(t)$ denote the Eulerian polynomials of type $A, B$ and $D$ respectively. For $A$ and $B$ it was showed by Barry $[2,3]$ who computed the Hankel transforms. Here we indicate the corresponding probability distributions $\mu_{t}^{\mathrm{A}}$, $\mu_{t}^{\mathrm{B}}$ and $\mu_{t}^{\mathrm{D}}$.

We are indebted to Philip B. Zhang for his helpful comments, which, in particular, led to Proposition 4.7.

\section{Eulerian numbers of type $A$}

The classical Eulerian numbers were introduced by Euler as a tool for studying infinite sums of the form (5). We define $A(n, k)$ as the number of permutations $\sigma \in \mathcal{S}_{n}$ having $k$ descents in the sequence $(\sigma(1), \ldots, \sigma(n))$. Here we present $A(n, k)$ for $0 \leqslant n \leqslant 4$ :

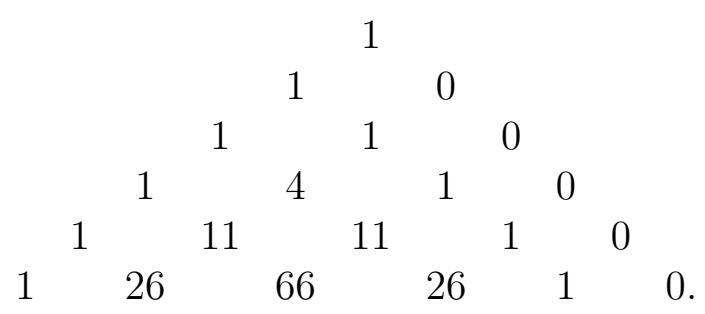

Let us record the main properties of $A(n, k)$, for details we refer to $[12,8]$ and to entry A123125 in OEIS. The numbers $A(n, k)$ admit the following recurrence relation:

$$
A(n, k)=(n-k) A(n-1, k-1)+(k+1) A(n-1, k)
$$

for $0<k<n$, with the boundary conditions: $A(n, 0)=1$ for $n \geqslant 0$ and $A(n, n)=0$ for $n \geqslant 1$. They also can be expressed in the following way:

$$
A(n, k)=\sum_{j=0}^{k}(-1)^{k-j}\left(\begin{array}{l}
n+1 \\
k-j
\end{array}\right)(j+1)^{n} .
$$

Let us also mention the Worpitzky formula: for $n \geqslant 0, x \in \mathbb{R}$ we have

$$
\sum_{k=0}^{n}\left(\begin{array}{c}
x+k \\
n
\end{array}\right) A(n, k)=x^{n} .
$$


The Eulerian polynomials are defined by

$$
P_{n}^{\mathrm{A}}(t):=\sum_{k=0}^{n} A(n, k) t^{k}
$$

in particular $P_{n}^{\mathrm{A}}(1)=n$ !. Euler himself encountered $P_{n}^{\mathrm{A}}(t)$ in the formula:

$$
\sum_{j=1}^{\infty} t^{j} j^{n}=\frac{t \cdot P_{n}^{\mathrm{A}}(t)}{(1-t)^{n+1}},
$$

which holds for $n \geqslant 0,|t|<1$. For $n \geqslant 1$ we have $A(n, k)=A(n, n-k-1)$, which implies

$$
t^{n-1} P_{n}^{\mathrm{A}}(1 / t)=P_{n}^{\mathrm{A}}(t) .
$$

The exponential generating function is equal to

$$
f^{\mathrm{A}}(t, z):=\sum_{n=0}^{\infty} \frac{P_{n}^{\mathrm{A}}(t)}{n !} z^{n}=\frac{t-1}{t-e^{(t-1) z}} .
$$

Recall that a sequence $\left\{a_{n}\right\}_{n=0}^{\infty}, a_{n} \in \mathbb{R}$, is said to be positive definite if the infinite matrix $\left(a_{i+j}\right)_{i, j=0}^{\infty}$ is positive definite, i.e. for every sequence $\left\{c_{i}\right\}_{i=0}^{\infty}, c_{i} \in \mathbb{R}$, with only finitely many nonzero entries, we have

$$
\sum_{i, j=0}^{\infty} a_{i+j} c_{i} c_{j} \geqslant 0
$$

This is equivalent to the fact, that there exists a nonnegative measure $\mu$ on $\mathbb{R}$ such that $a_{n}$ are moments of $\mu$, i.e. $a_{n}=\int x^{n} d \mu(x), n=0,1, \ldots$, see [1] for details.

It is well known, that $\left\{n !=P_{n}^{\mathrm{A}}(1)\right\}_{n=0}^{\infty}$ is positive definite as the moment sequence of the gamma distribution with weight $e^{-x}$ on $[0,+\infty)$. Barry $[2,3]$ showed that for $t \geqslant 0$ the sequence $\left\{P_{n}(t)\right\}_{n=0}^{\infty}$ is positive definite by computing the Hankel transform and Jacobi parameters. Here we are going to show, that for $0<t \neq 1, P_{n}(t)$ are moments of a dilated geometric distribution.

Theorem 2.1. If $t \geqslant 0$ then $\left\{P_{n}^{\mathrm{A}}(t)\right\}_{n=0}^{\infty}$ is the moment sequence of probability measure $\mu_{t}^{\mathrm{A}}$ given by: $\mu_{0}^{\mathrm{A}}=\delta_{1}$,

$$
\mu_{t}^{\mathrm{A}}=\sum_{j=1}^{\infty}(1-t) t^{j-1} \delta_{j(1-t)}
$$

if $0<t<1$,

$$
\mu_{1}^{\mathrm{A}}=e^{-x} \chi_{[0,+\infty)}(x) d x
$$

and for $t>1$

$$
\mu_{t}^{\mathrm{A}}=\sum_{j=0}^{\infty} \frac{t-1}{t^{j+1}} \delta_{j(t-1)} .
$$


Proof. We have to show that the $n$-th moment of $\mu_{t}^{\mathrm{A}}$ is $P_{n}^{\mathrm{A}}(t)$. For $0<t<1$ we have

$$
\int_{\mathbb{R}} t^{n} d \mu^{\mathrm{A}}(t)=\sum_{j=1}^{\infty}(1-t)^{n+1} j^{n} t^{j-1}
$$

which, in view of (5), is equal to $P_{n}^{\mathrm{A}}(t)$.

Assume that $t>1$. Then $\mu_{t}^{\mathrm{A}}(\mathbb{R})=1$ and for $n \geqslant 1$ we substitute $t:=1 / s$ :

$$
\int_{\mathbb{R}} t^{n} d \mu^{\mathrm{A}}(t)=\sum_{j=0}^{\infty} \frac{(t-1)^{n+1} j^{n}}{t^{j+1}}=\frac{(1-s)^{n+1}}{s^{n}} \sum_{j=1}^{\infty} j^{n} s^{j}=\frac{P_{n}^{\mathrm{A}}(s)}{s^{n-1}}
$$

and by (6) this is equal to $P_{n}^{\mathrm{A}}(1 / s)=P_{n}^{\mathrm{A}}(t)$.

Finally, by the definition of the gamma function we have

$$
\int_{0}^{+\infty} x^{n} e^{-x} d x=\Gamma(n+1)=n !=P_{n}^{\mathrm{A}}(1)
$$

which completes the proof.

\section{Eulerian numbers of type $B$}

We define $\mathcal{B}_{n}$ as the group of such permutations $\sigma$ of the set

$$
\{-n, \ldots,-1,0,1, \ldots, n\}
$$

that $\sigma$ is odd, i.e. $\sigma(-k)=-\sigma(k)$ for every $k,-n \leqslant k \leqslant n$. Then $\left|\mathcal{B}_{n}\right|=2^{n} n$ ! and $\mathcal{B}_{n}$ can be naturally viewed as a subgroup of the symmetric group $\mathcal{S}_{2 n}$. For $\sigma \in \mathcal{B}_{n}$ we denote by $\operatorname{desc}(\sigma)$ the number of descents in the sequence $(0, \sigma(1), \ldots, \sigma(n))$.

Denote by $\mathcal{B}_{n, k}$ the set $\left\{\sigma \in \mathcal{B}_{n}: \operatorname{desc}(\sigma)=k\right\}$, and by $B(n, k)$ its cardinality, $0 \leqslant k \leqslant$ n. $B(n, k)$ are called type $B$ Eulerian numbers. Here we record $B(n, k)$ for $0 \leqslant n \leqslant 4$ :

$\begin{array}{cccccccccc} & & & & 1 & & & & \\ & & & 1 & & 1 & & & \\ & & 1 & & 6 & & 1 & & \\ & 1 & & 23 & & 23 & & 1 & \\ & & 76 & & 230 & & 76 & & 1 .\end{array}$

Now we recall basic properties of $B(n, k)$, for details we refer to [5, 7] and to entry $A 060187$ in OEIS. First of all, they satisfy the recurrence relation:

$$
B(n, k)=(2 n-2 k+1) B(n-1, k-1)+(2 k+1) B(n-1, k),
$$

$0<k<n$, with the boundary conditions $B(n, 0)=B(n, n)=1$. Similarly to (2) and (3) we have equality

$$
B(n, k)=\sum_{j=0}^{k}(-1)^{k-j}\left(\begin{array}{l}
n+1 \\
k-j
\end{array}\right)(2 j+1)^{n},
$$


$0 \leqslant k \leqslant n$, and Worpitzky formula of the form:

$$
\sum_{k=0}^{n}\left(\begin{array}{c}
x+k \\
n
\end{array}\right) B(n, k)=(1+2 x)^{n}
$$

for $n \geqslant 0$.

The Eulerian polynomials of type $B$,

$$
P_{n}^{\mathrm{B}}(t):=\sum_{k=0}^{n} B(n, k) t^{k},
$$

are related to $P_{n}^{\mathrm{A}}(t)$ in the following way:

$$
(1+t)^{n+1} P_{n}^{\mathrm{A}}(t)-2^{n} t P_{n}^{\mathrm{A}}\left(t^{2}\right)=P_{n}^{\mathrm{B}}\left(t^{2}\right),
$$

see [13]. The symmetry $B(n, k)=B(n, n-k)$ implies $t^{n} \cdot P_{n}^{\mathrm{B}}(1 / t)=P_{n}^{\mathrm{B}}(t)$. For $|t|<1$, $n \geqslant 0$, we have odd version of $(5)$ :

$$
\sum_{k=0}^{\infty}(2 k+1)^{n} t^{k}=\frac{P_{n}^{\mathrm{B}}(t)}{(1-t)^{n+1}} .
$$

The exponential generating function is equal

$$
f^{\mathrm{B}}(t, z):=\sum_{n=0}^{\infty} \frac{P_{n}^{\mathrm{B}}(t)}{n !} z^{n}=\frac{(1-t) e^{(1-t) z}}{1-t e^{2(1-t) z}} .
$$

Similarly as for type $A$ we can prove that the sequence $\left\{P_{n}^{\mathrm{B}}(t)\right\}_{n=0}^{\infty}$ is positive definite for $t \geqslant 0$ (c.f. $[2,3])$ and indicate the corresponding probability measures.

Theorem 3.1. If $t \geqslant 0$ then $\left\{P_{n}^{\mathrm{B}}(t)\right\}_{n=0}^{\infty}$ is the moment sequence of probability measure $\mu_{t}^{\mathrm{B}}$ given by: $\mu_{0}^{\mathrm{B}}=\delta_{1}$,

$$
\mu_{t}^{\mathrm{B}}=\sum_{k=0}^{\infty}(1-t) t^{k} \delta_{(2 k+1)(1-t)}
$$

if $0<t<1$

$$
\mu_{1}^{\mathrm{B}}=\frac{1}{2} e^{-x / 2} \chi_{[0,+\infty)}(x) d x
$$

and for $t>1$

$$
\mu_{t}^{\mathrm{B}}=\sum_{k=0}^{\infty} \frac{t-1}{t^{k+1}} \delta_{(2 k+1)(t-1)} .
$$


Proof. The case $0<t<1$ is a consequence of (13). For $t>1$ we substitute $t:=1 / s$ and have

$$
\begin{gathered}
\int_{\mathbb{R}} t^{n} d \mu^{\mathrm{B}}(t)=\sum_{k=0}^{\infty} \frac{(t-1)^{n+1}(2 k+1)^{n}}{t^{k+1}} \\
=\frac{(1-s)^{n+1}}{s^{n}} \sum_{j=0}^{\infty}(2 k+1)^{n} s^{k}=\frac{P_{n}^{\mathrm{B}}(s)}{s^{n}}=P_{n}^{\mathrm{B}}(1 / s)=P_{n}^{\mathrm{B}}(t) .
\end{gathered}
$$

Finally

$$
\frac{1}{2} \int_{0}^{+\infty} x^{n} e^{-x / 2} d x=2^{n} n !=P_{n}^{\mathrm{B}}(1)
$$

\section{Eulerian numbers of type $D$}

By $\mathcal{D}_{n}$ we will denote the group of such elements $\sigma \in \mathcal{B}_{n}$ that the set $\{\sigma(1), \ldots, \sigma(n)\}$ contains an even number of negative terms. If $n \geqslant 1$ then $\mathcal{D}_{n}$ is a normal subgroup of $\mathcal{B}_{n}$ of index 2. Denote $\widetilde{\mathcal{D}}_{n}:=\mathcal{B}_{n} \backslash \mathcal{D}_{n}$ and

$$
\begin{aligned}
& \mathcal{D}_{n, k}:=\left\{\sigma \in \mathcal{D}_{n}: \operatorname{desc}(\sigma)=k\right\}, \\
& \widetilde{\mathcal{D}}_{n, k}:=\left\{\sigma \in \widetilde{\mathcal{D}}_{n}: \operatorname{desc}(\sigma)=k\right\},
\end{aligned}
$$

so that $\mathcal{D}_{n, k}=\mathcal{B}_{n, k} \cap \mathcal{D}_{n}, \widetilde{\mathcal{D}}_{n, k}=\mathcal{B}_{n, k} \backslash \mathcal{D}_{n}$. The cardinalities of these sets will be denoted $D(n, k)$ and $\widetilde{D}(n, k)$ respectively (A262226 and $A 262227$ in OEIS [10]). Since $\mathcal{B}_{n, k}=\mathcal{D}_{n, k} \dot{\cup} \widetilde{\mathcal{D}}_{n, k}$, we have

$$
B(n, k)=D(n, k)+\widetilde{D}(n, k) .
$$

Here we record the numbers $D(n, k)$ (the primary type $D$ triangle):

$\begin{array}{cccccccccc} & & & & 1 & & & & \\ & & & 1 & & 0 & & & \\ & & 1 & & 2 & & 1 & & \\ & & 1 & & 10 & & 13 & & 0 & \\ & & 36 & & 118 & & 36 & & 1\end{array}$

and $\widetilde{D}(n, k)$ (the complementary type $D$ triangle):

$\begin{array}{cccccccccc} & & & & 0 & & & & \\ & & & 0 & & 1 & & & \\ & & 0 & & 4 & & 0 & & \\ & 0 & & 13 & & 10 & & 1 & \\ 0 & & 40 & & 112 & & 40 & & 0\end{array}$

for $0 \leqslant n \leqslant 4$. In view of Proposition 4.7, every row in $D$ and in $\widetilde{D}$ constitutes a log-concave, and therefore unimodal sequence.

First we note the following symmetry. 
Proposition 4.1. For $0 \leqslant k \leqslant n$ we have

$$
D(n, k)=D(n, n-k), \quad \widetilde{D}(n, k)=\widetilde{D}(n, n-k)
$$

if $n$ is even and

$$
D(n, k)=\widetilde{D}(n, n-k), \quad \widetilde{D}(n, k)=D(n, n-k)
$$

if $n$ is odd.

Proof. For $\sigma \in \mathcal{B}_{n}$ define $-\sigma \in \mathcal{B}_{n}$ by $(-\sigma)(k):=-\sigma(k)$. It suffices to note that the map $\sigma \mapsto-\sigma$ defines bijections $\mathcal{D}_{n, k} \rightarrow \mathcal{D}_{n, n-k}, \widetilde{\mathcal{D}}_{n, k} \rightarrow \widetilde{\mathcal{D}}_{n, n-k}$ if $n$ is even and $\mathcal{D}_{n, k} \rightarrow \widetilde{\mathcal{D}}_{n, n-k}$, $\widetilde{\mathcal{D}}_{n, k} \rightarrow \mathcal{D}_{n, n-k}$ if $n$ is odd.

Now we present the fundamental recurrence relations for both triangles.

Theorem 4.2. The numbers $D(n, k), \widetilde{D}(n, k)$ admit the following recurrence relations:

$$
\begin{gathered}
D(n, k)=(k+1) D(n-1, k)+(n-k) D(n-1, k-1) \\
+k \widetilde{D}(n-1, k)+(n-k+1) \widetilde{D}(n-1, k-1)
\end{gathered}
$$

and

$$
\begin{gathered}
\widetilde{D}(n, k)=(k+1) \widetilde{D}(n-1, k)+(n-k) \widetilde{D}(n-1, k-1) \\
+k D(n-1, k)+(n-k+1) D(n-1, k-1)
\end{gathered}
$$

for $0<k<n$ and the boundary conditions: $D(n, 0)=1, \widetilde{D}(n, 0)=0$ and

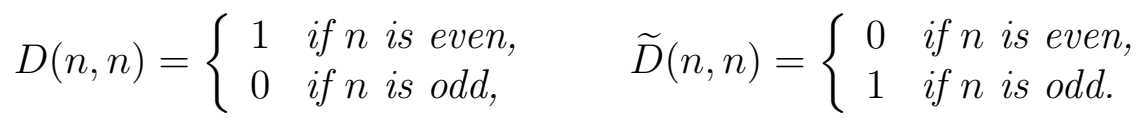

Proof. For the sake of this proof we will identify an element $\sigma \in \mathcal{B}_{n}$ with the sequence $\left(\sigma_{0}, \ldots, \sigma_{n}\right)$, where we write $\sigma_{k}$ instead of $\sigma(k)$. Now for $\left(\sigma_{0}, \ldots, \sigma_{n}\right) \in \mathcal{B}_{n}$ we define

$$
\Lambda \sigma:=\left(\sigma_{0}, \ldots, \widehat{\sigma}_{j}, \ldots, \sigma_{n}\right) \in \mathcal{B}_{n-1}
$$

where $j$ is such that $\sigma_{j}= \pm n$, and $\widehat{\sigma}_{j}$ means, that the element $\sigma_{j}$ has been removed from the sequence.

For given $\sigma \in \mathcal{D}_{n, k}, 0<k<n$, we have four possibilities:

- $\sigma_{j}=n$ and either $j=n$ or $\sigma_{j-1}>\sigma_{j+1}, 1 \leqslant j<n$. Then $\Lambda \sigma \in \mathcal{D}_{n-1, k}$.

- $\sigma_{j}=n$ and $\sigma_{j-1}<\sigma_{j+1}, 1 \leqslant j<n$. Then $\Lambda \sigma \in \mathcal{D}_{n-1, k-1}$.

- $\sigma_{j}=-n$ and $\sigma_{j-1}>\sigma_{j+1}, 1 \leqslant j<n$. Then $\Lambda \sigma \in \widetilde{\mathcal{D}}_{n-1, k}$.

- $\sigma_{j}=-n$ and either $j=n$ or $\sigma_{j-1}<\sigma_{j+1}, 1 \leqslant j<n$. Then $\Lambda \sigma \in \widetilde{\mathcal{D}}_{n-1, k-1}$. 
Now, suppose we are given a fixed $\tau=\left(\tau_{0}, \ldots, \tau_{n-1}\right)$ which belongs to one of the sets $\mathcal{D}_{n-1, k}, \mathcal{D}_{n-1, k-1}, \widetilde{\mathcal{D}}_{n-1, k}$ or $\widetilde{\mathcal{D}}_{n-1, k-1}$. We are looking for $\sigma \in \mathcal{D}_{n, k}$ such that $\Lambda \sigma=\tau$.

If $\tau \in \mathcal{D}_{n-1, k}$ then we should either put $n$ to the end of $\tau$, or insert $n$ into a drop of $\tau$, i.e. between $\tau_{i-1}$ and $\tau_{i}$, where $1 \leqslant i \leqslant n-1, \tau_{i-1}>\tau_{i}$, so we have $k+1$ possibilities.

Similarly, if $\tau \in \mathcal{D}_{n-1, k-1}$ then we construct $\sigma$ by inserting $n$ between $\tau_{i-1}$ and $\tau_{i}$, $1 \leqslant i \leqslant n-1$, where $\tau_{i-1}<\tau_{i}$. For this we have $n-k$ possibilities.

Now assume that $\tau \in \widetilde{\mathcal{D}}_{n-1, k}$. Now we should insert $-n$ between $\tau_{i-1}$ and $\tau_{i}, 1 \leqslant i \leqslant$ $n-1$, where $\tau_{i-1}>\tau_{i}$, for which we have $k$ possibilities.

Finally, if $\tau \in \widetilde{\mathcal{D}}_{n-1, k-1}$ then we put $-n$ either at the end of $\tau$ or between $\tau_{i-1}$ and $\tau_{i}$, $1 \leqslant i \leqslant n-1$, where $\tau_{i-1}<\tau_{i}$, for which we have $n-k+1$ possibilities.

Therefore the number of $\sigma \in \mathcal{D}_{n, k}$ such that $\Lambda \sigma$ belongs to the set $\mathcal{D}_{n-1, k}, \mathcal{D}_{n-1, k-1}$, $\widetilde{\mathcal{D}}_{n-1, k}$ or $\widetilde{\mathcal{D}}_{n-1, k-1}$ is equal to $(k+1) D(n-1, k),(n-k) D(n-1, k-1), k \widetilde{D}(n-1, k)$ or $(n-k+1) \widetilde{D}(n-1, k-1)$ respectively. This proves the recurrence for the numbers $D(n, k)$. For $\widetilde{D}(n, k)$ we proceed in the same way.

The boundary conditions are quite obvious: $(0,1,2, \ldots, n)$ is the only element $\sigma \in \mathcal{B}_{n}$ such that $\operatorname{desc}(\sigma)=0$ and $(0,-1,-2, \ldots,-n)$ is the only element $\tau \in \mathcal{B}_{n}$ with $\operatorname{desc}(\tau)=$ $n$. The former belongs to $\mathcal{D}_{n}$, while the latter belongs to $\mathcal{D}_{n}$ if and only if $n$ is even.

Now we show a simple relation between the numbers $D(n, k)$ and $\widetilde{D}(n, k)$.

Proposition 4.3. For $0 \leqslant k \leqslant n$ we have

$$
D(n, k)-\widetilde{D}(n, k)=(-1)^{k}\left(\begin{array}{l}
n \\
k
\end{array}\right) .
$$

Proof. For $k=0$ and $k=n$ this is straightforward. Assume that $0<k<n$ and that the equality holds for $n-1$. From Proposition 4.2 we have

$$
\begin{aligned}
D(n, k)- & \widetilde{D}(n, k)=(k+1)\left(\begin{array}{c}
n-1 \\
k
\end{array}\right)(-1)^{k}+(n-k)\left(\begin{array}{l}
n-1 \\
k-1
\end{array}\right)(-1)^{k-1} \\
& -k\left(\begin{array}{c}
n-1 \\
k
\end{array}\right)(-1)^{k}-(n-k+1)\left(\begin{array}{l}
n-1 \\
k-1
\end{array}\right)(-1)^{k-1} \\
= & \left(\begin{array}{c}
n-1 \\
k
\end{array}\right)(-1)^{k}-\left(\begin{array}{l}
n-1 \\
k-1
\end{array}\right)(-1)^{k-1}=\left(\begin{array}{l}
n \\
k
\end{array}\right)(-1)^{k} .
\end{aligned}
$$

Combining (15) with (16) we get

\section{Corollary 4.4.}

$$
\begin{aligned}
& D(n, k)=\frac{1}{2} B(n, k)+\frac{1}{2}\left(\begin{array}{l}
n \\
k
\end{array}\right)(-1)^{k}, \\
& \widetilde{D}(n, k)=\frac{1}{2} B(n, k)-\frac{1}{2}\left(\begin{array}{l}
n \\
k
\end{array}\right)(-1)^{k} .
\end{aligned}
$$


Now we are able to provide independent recurrences for $D(n, k)$ and $\widetilde{D}(n, k)$.

Proposition 4.5. The numbers $D(n, k), \widetilde{D}(n, k)$ admit the following recurrence:

$$
\begin{array}{ll}
D(n, 0)=1, & \widetilde{D}(n, 0)=0, \\
D(n, n)=\frac{1+(-1)^{n}}{2}, & \widetilde{D}(n, n)=\frac{1-(-1)^{n}}{2}
\end{array}
$$

and for $0<k<n$

$$
\begin{aligned}
& D(n, k)=(2 k+1) D(n-1, k)+(2 n-2 k+1) D(n-1, k-1)+\left(\begin{array}{l}
n-1 \\
k-1
\end{array}\right)(-1)^{k}, \\
& \widetilde{D}(n, k)=(2 k+1) \widetilde{D}(n-1, k)+(2 n-2 k+1) \widetilde{D}(n-1, k-1)-\left(\begin{array}{l}
n-1 \\
k-1
\end{array}\right)(-1)^{k} .
\end{aligned}
$$

Proof. This is a consequence of Proposition 4.2 and formula (16).

Now we can prove the following Worpitzky type identities.

Proposition 4.6. For $n \geqslant 0, x \in \mathbb{R}$ we have:

$$
\begin{aligned}
& 2 \sum_{k=0}^{n}\left(\begin{array}{c}
x+k \\
n
\end{array}\right) D(n, k)=(2 x+1)^{n}+(-1)^{n}, \\
& 2 \sum_{k=0}^{n}\left(\begin{array}{c}
x+k \\
n
\end{array}\right) \widetilde{D}(n, k)=(2 x+1)^{n}-(-1)^{n} .
\end{aligned}
$$

Proof. These formulas follow from Corollary 4.4, (10) and from the identity

$$
\sum_{k=0}^{n}\left(\begin{array}{c}
x+k \\
n
\end{array}\right)\left(\begin{array}{l}
n \\
k
\end{array}\right)(-1)^{k}=(-1)^{n},
$$

see for example (5.24) in [8].

Define polynomials

$$
\begin{aligned}
& P_{n}^{\mathrm{D}}(t):=\sum_{k=0}^{n} D(n, k) t^{k}, \\
& P_{n}^{\widetilde{\mathrm{D}}}(t):=\sum_{k=0}^{n} \widetilde{D}(n, k) t^{k} .
\end{aligned}
$$

It is known that for any finite irreducible Coxeter group the corresponding Eulerian polynomial has only real zeros, see $[5,13,14,11]$. It turns out to be also true for $P_{n}^{\mathrm{D}}(t)$ and $P_{n}^{\widetilde{\mathrm{D}}}(t)$.

Proposition 4.7. The polynomials $P_{n}^{\mathrm{D}}(t)$ and $P_{n}^{\widetilde{\mathrm{D}}}(t)$ have only real and simple zeros. 
Proof. This is a straightforward consequence of Corollary 6.9 in [4], where for $S$ we put the set of even or odd numbers in $\{1,2, \ldots, n\}$.

From (15) and (16) we have

$$
\begin{aligned}
& P_{n}^{\mathrm{D}}(t)+P_{n}^{\widetilde{\mathrm{D}}}(t)=P_{n}^{\mathrm{B}}(t), \\
& P_{n}^{\mathrm{D}}(t)-P_{n}^{\widetilde{\mathrm{D}}}(t)=(1-t)^{n},
\end{aligned}
$$

which implies

$$
\begin{aligned}
P_{n}^{\mathrm{D}}(t) & =\frac{1}{2} P_{n}^{\mathrm{B}}(t)+\frac{1}{2}(1-t)^{n}, \\
P_{n}^{\widetilde{\mathrm{D}}}(t) & =\frac{1}{2} P_{n}^{\mathrm{B}}(t)-\frac{1}{2}(1-t)^{n} .
\end{aligned}
$$

Proposition 4.8. For $n \geqslant 1$ we have

$$
\begin{aligned}
& P_{n}^{\mathrm{D}}(t)=(2 n t-t+1) P_{n-1}^{\mathrm{D}}(t)+2 t(1-t) \frac{\mathrm{d}}{\mathrm{d} t} P_{n-1}^{\mathrm{D}}(t)-t(1-t)^{n-1}, \\
& P_{n}^{\widetilde{\mathrm{D}}}(t)=(2 n t-t+1) P_{n-1}^{\widetilde{\mathrm{D}}}(t)+2 t(1-t) \frac{\mathrm{d}}{\mathrm{d} t} P_{n-1}^{\widetilde{\mathrm{D}}}(t)+t(1-t)^{n-1} .
\end{aligned}
$$

Proof. We have

$$
\begin{aligned}
\sum_{k=0}^{n}(2 k+1) D(n-1, k) t^{k} & =2 t \frac{\mathrm{d}}{\mathrm{d} t} P_{n-1}^{\mathrm{D}}(t)+P_{n-1}^{\mathrm{D}}(t), \\
\sum_{k=0}^{n}(2 n-2 k+1) D(n-1, k-1) t^{k} & =(2 n-1) t P_{n-1}^{\mathrm{D}}(t)-2 t^{2} \frac{\mathrm{d}}{\mathrm{d} t} P_{n-1}^{\mathrm{D}}(t)
\end{aligned}
$$

and

$$
\sum_{k=0}^{n}\left(\begin{array}{l}
n-1 \\
k-1
\end{array}\right)(-1)^{k} t^{k}=-t(1-t)^{n-1}
$$

Summing up and applying Proposition 4.5 we get the first equality. The second one we obtain in the same way.

Now we are able to find the generating functions.

Proposition 4.9. The exponential generating functions

$$
\begin{aligned}
f^{\mathrm{D}}(t, z) & :=\sum_{n=0}^{\infty} \frac{P_{n}^{\mathrm{D}}(t)}{n !} z^{n}, \\
f^{\widetilde{\mathrm{D}}}(t, z) & :=\sum_{n=0}^{\infty} \frac{P_{n}^{\widetilde{\mathrm{D}}}(t)}{n !} z^{n}
\end{aligned}
$$


satisfy the following differential equations

$$
\begin{gathered}
(1+t) f^{\mathrm{D}}(t, z)+(2 t z-1) \frac{\partial f^{\mathrm{D}}}{\partial z}(t, z)+2 t(1-t) \frac{\partial f^{\mathrm{D}}}{\partial t}(t, z)=t e^{(1-t) z} \\
(1+t) f^{\widetilde{\mathrm{D}}}(t, z)+(2 t z-1) \frac{\partial f^{\widetilde{\mathrm{D}}}}{\partial z}(t, z)+2 t(1-t) \frac{\partial f^{\widetilde{\mathrm{D}}}}{\partial t}(t, z)=-t e^{(1-t) z},
\end{gathered}
$$

with the initial conditions $f^{\widetilde{\mathrm{D}}}(t, 0)=1, f^{\widetilde{\mathrm{D}}}(t, 0)=0$. Moreover,

$$
\begin{aligned}
f^{\mathrm{D}}(t, z) & =\frac{(2-t) e^{(1-t) z}-t e^{3(1-t) z}}{2-2 t e^{2(1-t) z}}, \\
f^{\widetilde{\mathrm{D}}}(t, z) & =\frac{t e^{3(1-t) z}-t e^{(1-t) z}}{2-2 t e^{2(1-t) z}} .
\end{aligned}
$$

Proof. Multiplying both sides of (26) by $z^{n} / n$ ! and taking sum $\sum_{n=0}^{\infty}$ we obtain the equation

$$
f^{\mathrm{D}}=2 t z f^{\mathrm{D}}+(1-t) F^{\mathrm{D}}+2 t(1-t) \frac{\partial F^{\mathrm{D}}}{\partial t}-\frac{t}{1-t} e^{(1-t) z},
$$

where

$$
F^{\mathrm{D}}(t, z):=\sum_{n=1}^{\infty} \frac{P_{n-1}^{\mathrm{D}}(t)}{n !} z^{n} .
$$

Taking the derivative $\frac{\partial}{\partial z}$ of both sides of $(30)$ and keeping in mind that $\frac{\partial F^{\mathrm{D}}}{\partial z}=f^{\mathrm{D}}$, we get the first differential equation. The second is obtained in the same way.

Formulas $(28,29)$ follow directly from $(14)$ and $(24,25)$.

Finally we prove positive definiteness of the sequence $\left\{P_{n}^{\mathrm{D}}(t)\right\}_{n=0}^{\infty}$.

Theorem 4.10. If $t \geqslant 0$ then $\left\{P_{n}^{\mathrm{D}}(t)\right\}_{n=0}^{\infty}$ is the moment sequence of probability measure $\mu_{t}^{\mathrm{D}}$ given by: $\mu_{0}^{\mathrm{D}}=\delta_{1}$,

$$
\mu_{t}^{\mathrm{D}}=\frac{2-t}{2} \delta_{1-t}+\sum_{k=1}^{\infty} \frac{(1-t) t^{k}}{2} \delta_{(2 k+1)(1-t)}
$$

if $0<t<1$

$$
\mu_{1}^{\mathrm{D}}=\frac{1}{2} \delta_{0}+\frac{1}{4} e^{-x / 2} \chi_{[0,+\infty)}(x) d x
$$

and for $t>1$

$$
\mu_{t}^{\mathrm{D}}=\frac{1}{2} \delta_{1-t}+\sum_{k=0}^{\infty} \frac{t-1}{2 t^{k+1}} \delta_{(2 k+1)(t-1)} .
$$

Proof. From (24) we have $\mu_{t}^{\mathrm{D}}=\frac{1}{2} \mu_{t}^{\mathrm{B}}+\frac{1}{2} \delta_{1-t}$ and now we apply Theorem 3.1. 
Since $\left\{P_{n}^{\widetilde{\mathrm{D}}}(t)\right\}_{n=0}^{\infty}$ is the moment sequence of the non-positive measure $\frac{1}{2} \mu_{t}^{\mathrm{B}}-\frac{1}{2} \delta_{1-t}$, it is not positive definite. It turns out however, that for $t \geqslant 1$ the sequence $\left\{P_{n+1}^{\widetilde{\mathrm{D}}}(t)\right\}_{n=0}^{\infty}$ is positive definite.

Proposition 4.11. For $t \geqslant 1$ the sequence $\left\{P_{n+1}^{\widetilde{\mathrm{D}}}(t) / t\right\}_{n=0}^{\infty}$ is positive definite and the corresponding probability measure $\nu_{t}$ is

$$
\nu_{1}=\frac{1}{4} x e^{-x / 2} \chi_{[0,+\infty)}(x) d x
$$

and for $t>1$

$$
\nu_{t}=\frac{t-1}{2 t} \delta_{1-t}+\sum_{k=0}^{\infty} \frac{(t-1)^{2}(2 k+1)}{2 t^{k+2}} \delta_{(t-1)(2 k+1)} .
$$

Proof. We have

$$
P_{n+1}^{\widetilde{\mathrm{D}}}(t)=\int x^{n} \cdot x d\left(\frac{1}{2} \mu_{t}^{\mathrm{B}}-\frac{1}{2} \delta_{1-t}\right)(x)
$$

and the measure

$$
\nu_{t}=\frac{x}{t} d\left(\frac{1}{2} \mu_{t}^{\mathrm{B}}-\frac{1}{2} \delta_{1-t}\right)(x)
$$

is positive for $t \geqslant 1$.

\section{References}

[1] N. I. Akhiezer, The classical moment problem and some related questions in analysis, translated from the Russian by N. Kemmer, Hafner Publishing Co., New York 1965.

[2] P. Barry, Eulerian polynomials as moments, via exponential Riordan arrays, J. Integer Seq. 14 (2011), Article 11.9.5.

[3] P. Barry, General Eulerian polynomials as moments using exponential Riordan arrays, J. Integer Seq. 16 (2013), Article 13.9.6.

[4] P. Brändén, On linear transformations preserving the Pólya frequency property, Trans. Amer. Math. Soc. 358 no. 8 (2006) 3697-3716.

[5] F. Brenti, q-Eulerian polynomials arising from Coxeter groups, European J. Combin. 15 (1994) 417-441.

[6] Chak-On Chow, On the Eulerian polynomials of type D, European J. Combin. 24 (2003) 391-408.

[7] Chak-On Chow and I. M. Gessel, On the descent numbers and major indices for the hyperoctahedral group, Adv. Appl. Math. 38 no. 3 (2007) 275-301.

[8] R. L. Graham, D. E. Knuth and O. Patashnik, Concrete Mathematics. A Foundation for Computer Science, Addison-Wesley, New York, 1994. 
[9] M. Hyatt, Recurrences for Eulerian polynomials of type $B$ and type $D$, arXiv:1404.3110 (2014).

[10] N. J. A. Sloane, The On-line Encyclopedia of Integer Sequences, http://oeis.org/.

[11] C. D. Savage and M. Visontai, The s-Eulerian polynomials have only real roots, Trans. Amer. Math. Soc. 367 no. 2 (2015) 1441-1466.

[12] R. P. Stanley, Enumerative Combinatorics, vol. 1, Cambridge University Press, Cambridge, New York, 1997.

[13] A. L. B. Yang and P. B. Zhang, The real-rootedness of Eulerian polynomials via the Hermite-Biehler theorem, A Post Presentation in FPSAC 2015, arXiv:1501.05824 (2015).

[14] P. B. Zhang, On the real-rootedness of the descent polynomials of $(n-2)$-stack sortable permutations, Electron. J. Combin. 22 (4) (2015) \#P4.12. 